\title{
Phase Behavior of Magnetic Colloid-Polymer Mixtures: 1. Mean Field Calculations
}

\author{
G. A. van Ewijk and G. J. Vroege* \\ Van't Hoff Laboratory for Physical and Colloid Chemistry, Debye Institute, Utrecht University, \\ Padualaan 8, $3584 \mathrm{CH}$ Utrecht, The Netherlands
}

Received J uly 17, 2001. In Final Form: October 9, 2001

\begin{abstract}
The phase behavior of mixtures of magnetic colloids and a nonadsorbing polymer has been investigated using mean field theory. The polymer in this mixture causes an effective isotropic attraction (depletion attraction) between the magnetic colloids, the range and strength of which can be varied independently from the (anisotropic) dipole-dipole interaction. Calculations within this mean field approximation show that in the absence of a magnetic field a magnetic colloid-polymer mixture may phase separate into a polymer-rich colloidal gas and a polymer-poor colloidal liquid. Upon application of a magnetic field, the minimum amount of polymer needed to destabilize the mixture is reduced. However, for a dipole-dipole interaction strength typical for real ferrofluids, this reduction is insufficient to give phase coexistence in a polymer-freeferrofluid. Thetheory al so suggests that freeol eicacid, which is present in many ferrofluids, may decrease a ferrofluid's stability in the same way that a polymer does.
\end{abstract}

\section{Introduction}

Ferrofluids are colloidal dispersions of ferro- or ferrimagnetic particles in a nonmagnetic solvent. ${ }^{1}$ An example of such particles is spherical magnetite $\left(\mathrm{Fe}_{3} \mathrm{O}_{4}\right)$ particles with a typical size of $10 \mathrm{~nm}$, which are present in many ferrofluids. Because of its monodomain nature, each particle has a permanent magnetic dipole moment. The macroscopic properties of ferrofluids are therefore frequently compared with dipolar hard sphere (DHS) fluid theories.

Theoretical predictions on the phase behavior of DHS fluids are still controversial.2,3 On one hand, various theories such as mean field theories, ${ }^{4-6}$ mean spherical approximation, ${ }^{7}$ and perturbation theories ${ }^{8}$ predict the existence of a gas-liquid equilibrium even in the absence of a magnetic field. On the other hand, computer simulations ${ }^{9,10}$ fail to find such an equilibrium, except when isotropic attraction is present in addition to dipolar interaction. ${ }^{9}$

Experimentally, colloidal gas-liquid phase separation has been observed in ferrofluids under various conditions. ${ }^{11}$ For example, application of a magnetic field to a stable ferrofluid can induce the formation of concentrated droplets in a dilute background. ${ }^{12-16}$ In zerofiel d, charge-

* Corresponding author. E-mail: g.j.vroege@chem.uu.nl.

(1) Rosensweig, R. E. Ferrohydrodynamics; Cambridge University Press: Cambridge, 1985.

(2) Teixeira, P. I. C.; Tavares, J . M.; Telo da Gama, M. M. J . Phys. 2000, 12, R411.

(3) Tlusty, T.; Safran, S. A. Science 2000, 290, 1328

(4) Tsebers, A. O. Magnetohydrodynamics 1982, 18, 137.

(5) Sano, K.; Doi, M. J . Phys. Soc. J pn. 1983, 52, 2810.

(6) Zhang, H.; Widom, M. Phys. Rev. E 1994, 49, 3591.

(7) Morozov, K. I.; Pshenichnikov, A. F.; Raikher, Y. I.; Shliomis, M.

I. J . Magn. Magn. Mater. 1987, 65, 269.

(8) Buyevich, Y. A.; Ivanov, A. O. Physica A 1992, 190, 276.

(9) van Leeuwen, M. E.; Smit, B. Phys. Rev. Lett. 1993, 71, 3991.

(10) Weis, J. J .; Levesque, D. Phys. Rev. Lett. 1993, 71, 2729.

(11) Cabuil, V. Curr. Opin. Colloid Interface Sci. 2000, 5, 44.

(12) Pshenichnikov, A. F.; Shurubor, I. Y. Izv. Akad. Nauk SSSR, Ser. Fiz. 1987, 51, 1081.

(13) Rosensweig, R. E.; Popplewell, J . In ElectromagneticF orces and Applications; Tani, J ., Takagi, T., Eds.; Elsevier Science Publishers: Amsterdam, 1992; p 83.

(14) Wang, H.; Zhu, Y.; Boyd, C.; Luo, W.; Cebers, A.; Rosensweig, R. E. Phys. Rev. Lett. 1994, 72, 1929. stabilized magnetic fluids become thermodynamically unstable when the ionic strength exceeds a critical value. ${ }^{17-19}$ Sterically stabilized magnetic colloids can be made to phase separate by reducing the solvent quality ${ }^{20}$ or adding a nonadsorbing polymer. ${ }^{21}$ All instabilities in zerofiel d probably involvea significant amount of isotropic attraction, in the form of either van der Waals attraction in aqueous ferrofluids ${ }^{19}$ and ferrofluids in a poor solvent or depletion attraction in mixtures of magnetic particles and free polymer. ${ }^{21}$ Clearly, the DHS description of ferrofluids is insufficient todescribetheobserved behavior.

In this paper, a mean fiel d theory is described which is used to predict the phase behavior of ferrofluids containing free polymer. The polymer induces an effectiveattraction (depletion attraction) between themagneticparticles with a strength proportional to the polymer concentration and a range comparable to the size of the polymer coils.22,23 Thus depletion attraction provides a means to vary isotropic attraction independently from magnetic interaction, making it possibletoexperimentally study theeffects of both types of interaction on the phase behavior of ferrofluids. In an accompanying paper ${ }^{24}$ we report on an experimental study of a ferrofluid-polymer mixture.

The theory described here is a combination of the "free volume approach" for colloid-polymer mixtures ${ }^{25}$ and perturbation theory for DHS fluids. ${ }^{8}$ Calculations on

(15) Hong, C.-Y.; J ang, I. J .; Horng, H. E.; Hsu, C. J .; Yao, Y. D. Yang, H. C. J . Appl. Phys. 1997, 81, 4275.

(16) Horng, H.-E.; Hong, C.-Y.; Yeung, W. B.; Yang, H.-C. Appl . Opt. 1998, 37, 2674

(17) Bacri, J .-C.; Perzynski, R.; Salin, D.; Cabuil, V.; Massart, R. J Colloid Interface Sci. 1988, 132,43.

(18) Cabuil, V.; Dubois, E.; Neveu, S.; Bacri, J .-C.; Hasmonay, E.; Perzynski, R. Prog. Colloid Polym. Sci. 1995, 98, 23.

(19) Dubois, E.; Perzynski, R.; Boué, F.; Cabuil, V. Langmuir 2000, 16,5617

(20) Dubois, E.; Cabuil, V.; Boué, F .; Bacri, J .-C.; Perzynski, R. Prog. Colloid Polym. Sci. 1997, 104, 1173.

(21) Cabuil, V.; Perzynski, R.; Bastide, J . Prog. Colloid Polym. Sci. $1994,97,75$

(22) Asakura, S.; Oosawa, F. J . Chem. Phys. 1954, 22, 1255

(23) Vrij, A. Pure Appl. Chem. 1976, 48, 471.

(24) van Ewijk, G. A.; Vroege, G. J .; Kuipers, B. W. M. Langmuir 2001, 18, 382.

(25) Lekkerkerker, H. N.W.; Poon, W. C.-K.; Pusey, P. N.; Stroobants, A.; Warren, P. B. Europhys. Lett. 1992, 20, 559. 


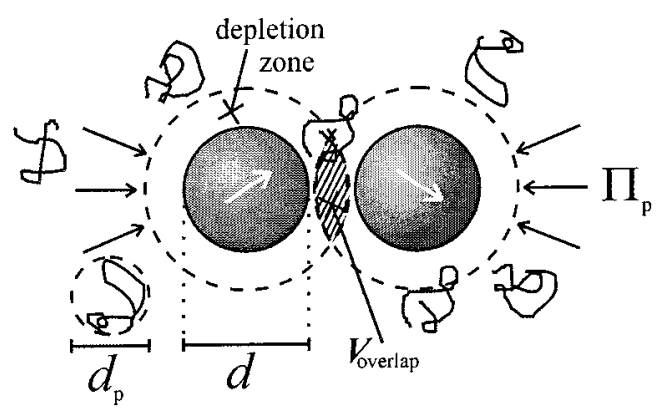

Figure 1. Two magnetic colloids at close distance experience an effective isotropic attraction, induced by the surrounding polymers.

systems with a magnetic interaction strength comparable to or somewhat higher than that in commonly used oleic acid stabilized ferrofluids show that field-induced phase separation does not occur in such ferrofluids, contrary to several reported experimental findings ${ }^{12-16}$ is in agreement with our experimental results on a ferrofluid from which clusters and free oleic acid had been removed. ${ }^{24}$ With sufficient free polymer present, the system exhibits gas-liquid separation even in zerofield. M oreover, phase separation is promoted by application of a magnetic field. As a final note, it is shown that removing free surfactant from oleicacid-stabilized ferrofluids may provebeneficial for the stability of these ferrofluids.

\section{Theory}

The principle of depletion attraction can beunderstood by considering two colloidal spheres with diameter $d$, immersed in a solution containing polymer coils with diameter $d_{p}$. The polymers are treated here as being interpenetrable with respect to each other, but hard repulsive toward the colloids. Therefore, each colloid is surrounded by a "depleti on zone" which is free of polymer. If the colloids are at a distance at which their depletion zones overlap (Figure 1), polymer is absent between the colloids, resulting in an osmotic pressure difference, $\Pi_{p}$, pushing these colloids together. This effective attraction has a range of $\left(d+d_{p}\right)$ and a minimum energy of $-\Pi_{p} V_{\text {overlap}}$, thus depending on the polymer concentration.

In this paper, the phase behavior of mixtures of magnetic colloids and polymer is considered. Therefore, the interaction between the magnetic dipole moments $\mathbf{m}$ embedded in the colloids and the interaction of these dipoles with an external magnetic field $\mathbf{H}$ must also be taken intoaccount. Thetotal potential energy of thesystem containing $\mathrm{N}$ colloidal particles and $\mathrm{N}_{\mathrm{p}}$ polymer coils is

$$
\begin{aligned}
U=\frac{1}{2} \sum_{i}^{N} \sum_{j \neq i}^{N} u_{h s}\left(r_{i j}\right)+\frac{1}{2} \sum_{i}^{N} \sum_{j \neq i}^{N} u_{d d}\left(r_{i j}, \Omega_{i}, \Omega_{j}\right)+ & + \\
& \sum_{i}^{N} u_{m}\left(\Omega_{i}\right)+\sum_{i}^{N} \sum_{j}^{N} u_{c p}\left(\tilde{r}_{i j}\right)
\end{aligned}
$$

where $u_{\mathrm{hs}}\left(r_{\mathrm{ij}}\right)$ is the hard-sphere pair potential between two colloids at distance $r_{\mathrm{ij}}$ and $\mathrm{u}_{\mathrm{dd}}\left(\mathbf{r}_{\mathrm{ij}}, \Omega_{\mathrm{i}}, \Omega_{\mathrm{j}}\right)$ is the interaction between two dipoles $\mathbf{m}_{\mathrm{i}}=\mathbf{m}\left(\Omega_{\mathrm{i}}\right)$ and $\mathbf{m}_{\mathrm{j}}=\mathbf{m}\left(\Omega_{\mathrm{j}}\right)$ connected by the vector $\mathbf{r}_{\mathrm{ij}}=\mathbf{r}_{\mathrm{j}}-\mathbf{r}_{\mathrm{i}}$

$$
\begin{gathered}
\beta \mathrm{u}_{\mathrm{dd}}\left(\mathbf{r}_{\mathrm{ij}}, \Omega_{\mathrm{i}}, \Omega_{\mathrm{j}}\right)=-\lambda \frac{\mathrm{d}^{3}}{\mathrm{r}_{\mathrm{ij}}{ }^{3}}\left[3\left(\hat{\mathrm{m}}_{\mathrm{i}} \cdot \hat{\mathrm{r}}_{\mathrm{ij}}\right)\left(\hat{\mathrm{m}}_{\mathrm{j}} \cdot \hat{\mathrm{r}}_{\mathrm{ij}}\right)-\hat{\mathrm{m}}_{\mathrm{i}} \cdot \hat{\mathrm{m}}_{\mathrm{j}}\right] \\
\lambda=\beta \frac{\mu_{0} \mathrm{~m}^{2}}{4 \pi \mathrm{d}^{3}}
\end{gathered}
$$

where $\beta=(\mathrm{kT})^{-1}, \lambda$ is a dimensionless coupling constant measuring the dipole-dipole interaction strength, $\mu_{0}$ is the permeability of vacuum, and $m$ is the dipol e moment. Circumflexes denote unit vectors. The interaction of a dipole with an external field is described by

$$
\beta \mathrm{u}_{\mathrm{m}}\left(\Omega_{\mathrm{i}}\right)=-\alpha \hat{\mathbf{m}}_{\mathrm{i}} \cdot \hat{\mathbf{H}} ; \quad \alpha=\beta \mu_{0} \mathrm{mH}
$$

Note that the expressions for $\lambda$ and $\alpha$ in eqs (2) and (3) are specific for the SI unit system. The remainder of this section is independent of the chosen unit system.

The polymer coils are regarded as nonadditive hard spheres, i.e., they experience no interaction with other polymer coils, but behaveas hard spheres toward colloids. This colloid-polymer interaction is given by $u_{\mathrm{cp}}\left(\tilde{r}_{\mathrm{ij}}\right)$ and depends on the colloid-polymer distance $\tilde{r}_{i j}=\left|\tilde{\mathbf{r}}_{\mathrm{j}}-\mathbf{r}_{\mathrm{i}}\right|$.

The phase diagram of the magnetic colloid-polymer mixture is calculated under constant volume restriction. The configuration integral for the system is

$$
\begin{gathered}
\mathrm{Z}=\int . \cdot \int \exp \left[-\beta \mathrm{U}\left(\mathbf{r}^{\mathrm{N}}, \Omega^{\mathrm{N}}, \tilde{\mathbf{r}}^{\mathrm{N}_{\mathrm{p}}}\right)\right] \mathrm{d} \mathbf{r}^{\mathrm{N}} \mathrm{d} \Omega^{\mathrm{N}} \mathrm{d} \tilde{\mathbf{r}}^{\mathrm{N}_{\mathrm{p}}} \\
\mathrm{d} \Omega_{\mathrm{i}}=(4 \pi)^{-1} \sin \theta_{\mathrm{i}} \mathrm{d} \theta_{\mathrm{i}} \mathrm{d} \phi_{\mathrm{i}}
\end{gathered}
$$

Because of the nonadditivity of the polymer coils, each integral over a coil center $\tilde{\mathbf{r}}_{\mathbf{i}}$ can beevaluated individually, each giving the vol ume accessibleto a pol ymer at a certain magnetic colloid configuration, $\mathrm{V}_{\text {free }}\left(\mathbf{r}^{\mathrm{N}}\right)$. As an approximation, the configuration-dependent $V_{\text {free }}\left(\mathbf{r}^{N}\right)$ is replaced by its mean value $\left\langle\mathrm{V}_{\text {free }}\right\rangle_{0}$, calculated by averaging over all configurations of a pure hard sphere fluid. Thus the influence of polymers and dipolar interactions on the free volume is neglected. With this, eq 4 becomes

$$
\begin{aligned}
\mathrm{Z}=[\mathrm{fV}]^{\mathrm{N}_{\mathrm{p}}} \int \cdot . \int \exp \left[-\beta \frac{1}{2} \sum_{\mathrm{i}, \mathrm{j} \neq \mathrm{i}}^{\mathrm{N}} \mathrm{u}_{\mathrm{hs}}\left(\mathrm{r}_{\mathrm{ij}}\right)-\right. \\
\left.\quad \beta \frac{1}{2} \sum_{\mathrm{i}, \mathrm{j} \neq \mathrm{i}}^{\mathrm{N}} \mathrm{u}_{\mathrm{dd}}\left(\mathbf{r}_{\mathrm{ij}}, \Omega_{\mathrm{i}}, \Omega_{\mathrm{j}}\right)-\beta \sum_{\mathrm{i}}^{\mathrm{N}} \mathrm{u}_{\mathrm{m}}\left(\Omega_{\mathrm{i}}\right)\right] \mathrm{d} \mathbf{r}^{\mathrm{N}} \mathrm{d} \Omega^{\mathrm{N}}
\end{aligned}
$$

wherethe integral in (5) is the configuration integral $Z_{\mathrm{DHS}}$ of a pure dipolar hard sphere system and $\mathrm{fV}=\left\langle\mathrm{V}_{\text {free }}\right\rangle_{0}$, so fis the fraction of theentirevolume $V$ accessibletopolymer. TheHelmholtzfreeenergy $F=F$ id $-k T \ln \left(Z N N^{N+N_{p}}\right)$ derived from (5) is now the sum of a dipolar hard sphere part, $\mathrm{F}_{\mathrm{DHS}}$, and a polymer part, $\mathrm{F}_{\mathrm{p}}$

$$
F=F_{D H S}+N_{p} k T \ln \frac{\phi_{p}}{f}
$$

where $\phi_{p}$ is the polymer "volume fraction" $N_{p}(\pi / 6) d_{p}{ }^{3} N$. The free volume fraction $f$ can be obtained by using the scaled particle theory, ${ }^{26}$ yiel ding

$$
\begin{aligned}
f=\left(1-\phi_{c}\right) \times & \quad \exp \left[-A\left(\frac{\phi_{c}}{1-\phi_{c}}\right)-B\left(\frac{\phi_{c}}{1-\phi_{c}}\right)^{2}-C\left(\frac{\phi_{c}}{1-\phi_{c}}\right)^{3}\right]
\end{aligned}
$$

where $\phi_{c}=N(\pi / 6) d^{3} N$ is the colloid volume fraction, $A=$ $3 q+3 q^{2}+q^{3}, B=9 q^{2} / 2+3 q^{3}, C=3 q^{3}$, and $q$ is the polymer-to-colloid size ratio: $q=d_{p} / d$.

Thefreeenergy of dipolar hard spheres has been derived via various routes, $4,8,27-29$ but most of them are not

(26) Lebowitz, J . L.; Helfand, E.; Praestgaard, E. J . Chem. Phys. 1965, 43, 774.

(27) Sutherland, J. W. H.; Nienhuis, G.; Deutch, J . M. Mol. Phys. 1974, 27, 721.

(28) Woodward, C. E.; Nordholm, S. Mol. Phys. 1984, 52, 973. 
applicable to magnetized systems. Because we are interested in the effect of a magnetic field, our choice is limited to two theories: the self-consistent field theory ${ }^{4}$ and the perturbation theory. ${ }^{8}$ The former is well-known in solidstate physics but has the questionable property of predicting spontaneous magnetization, even at low concentration or moderate interaction strength. Up to now, spontaneously magnetized ferrofluids have not been observed experimentally, and computer simulations only find spontaneous magnetization at high densities and strong interaction (e.g., $\phi_{\mathrm{c}}=0.42$ and $\lambda=5.3$ for dipolar hard spheres, ${ }^{30} \phi_{\mathrm{c}}=0.31$ and $\lambda=6.7$ for dipolar soft spheres ${ }^{31}$ ). Moreover, the concentration dependence of the magneticsusceptibility of ferrofluids deviates significantly from the susceptibility predicted by the self-consistent field theory. ${ }^{32-34}$ Predictions donewith perturbation theory are in better agreement with the measurements. We ther efore choose to use this theory, despite its limitation to weak interaction strength.

If dipole-dipole interactions are weak compared to $\mathrm{kT}$, the part of $\mathrm{Z}_{\mathrm{DHS}}$ containing dipole-dipoleinteractions can be rewritten in terms of Mayer functions $\Phi_{\mathrm{dd}}(\mathrm{ij})=$ $\exp \left[-\beta \mathrm{u}_{\mathrm{dd}}\left(\mathbf{r}_{\mathrm{ij}}, \Omega_{\mathrm{i}}, \Omega_{\mathrm{j}}\right)\right]-1$ and the product of all $\left(\Phi_{\mathrm{dd}}(\mathrm{ij})+1\right)$ can be expanded

$$
\begin{array}{r}
\mathrm{Z}_{\text {DHS }}=\int . . \int \exp \left[-\beta \frac{1}{2} \sum_{\mathrm{i}, \mathrm{j \neq i}}^{\mathrm{N}} \mathrm{u}_{\mathrm{hs}}\left(\mathrm{r}_{\mathrm{ij}}\right)-\beta \sum_{\mathrm{i}}^{\mathrm{N}} \mathrm{u}_{\mathrm{m}}\left(\Omega_{\mathrm{i}}\right)\right] \times \\
{\left[1+\frac{1}{2} \sum_{\mathrm{i}, \mathrm{j} \neq \mathrm{i}}^{\mathrm{N}} \Phi_{\mathrm{dd}}(\mathrm{ij})+\ldots\right] \mathrm{d} \mathbf{r}^{\mathrm{N}} \mathrm{d} \Omega^{\mathrm{N}}}
\end{array}
$$

Since the integration volumes are the same for all dipole pairs, thesum of Mayer functions gives $\mathrm{N}(\mathrm{N}-1)$ identical terms $\Phi_{\text {dd }}(12)$ hence integration over the orientations $\Omega_{3}$ to $\Omega_{\mathrm{N}}$ can be carried out immediately, resulting in one term $\sinh (\alpha) / \alpha$ for each dipole. By integration over the positions $\mathbf{r}_{3}$ to $\mathbf{r}_{N}$, the (hard sphere) pair distribution function $\mathrm{g}^{2}\left(\mathbf{r}_{12}\right)^{35}$ can be introduced into (8)

$$
\begin{gathered}
\mathrm{Z}_{\mathrm{DHS}}=\mathrm{Z}_{\mathrm{HS}}\left(\frac{\sinh \alpha}{\alpha}\right)^{\mathrm{N}}\left[1+\mathrm{N} \phi_{\mathrm{c}} \mathrm{G}(\mathrm{T}, \mathrm{H})\right] \\
\mathrm{G}(\mathrm{T}, \mathrm{H})=\frac{1}{2 \mathrm{v}_{\mathrm{c}}}\left(\frac{\alpha}{\sinh \alpha}\right)^{2} \int \cdot . \int \Phi_{\mathrm{dd}}(12) \mathrm{g}^{(2)}\left(\mathbf{r}_{12}\right) \times \\
\quad \exp \left[-\beta \mathrm{u}_{\mathrm{m}}\left(\Omega_{1}\right)-\beta \mathrm{u}_{\mathrm{m}}\left(\Omega_{2}\right)\right] \mathrm{d} \mathbf{r}_{12} \mathrm{~d} \Omega_{1} \mathrm{~d} \Omega_{2}
\end{gathered}
$$

where $v_{c}=(\pi / 6) d^{3}$ is the volume of a magnetic particle.

Combined with (6), eq 9 gives the total free energy of the magnetic colloid-polymer mixture

$$
\begin{aligned}
& \mathrm{F}=\mathrm{F}_{\mathrm{HS}}+\mathrm{N}_{\mathrm{p}} \mathrm{kT} \ln \frac{\phi_{\mathrm{p}}}{\mathrm{f}}-\mathrm{NkT} \ln \frac{\sinh \alpha}{\alpha}- \\
& \mathrm{NkT} \phi_{\mathrm{c}} \mathrm{G}(\mathrm{T}, \mathrm{H})
\end{aligned}
$$

To calculate $G(T, H)$, the Mayer function is Taylor expanded and only the linear and quadratic term are

(29) Berkoswky, B. M.; Kalikmanov, V. I.; Filinov, V. S. J . Magn. Magn. Mater. 1987, 65, 191.

(30) Weis, J .J .; Levesque, D.; Zarragoi coechea, G. J . Phys. Rev. Lett. 1992, 69, 913.

(31) Wei, D.; Patey, G. N. Phys. Rev. Lett. 1992, 68, 2043.

(32) van E wijk, G. A. Phasebehavior of mixtures of magnetic colloids and nonadsorbing polymer. Ph.D. Thesis, Utrecht University, 2001.

(33) Pshenichnikov, A. F.; Lebedev, A. V.; Morozov, K. I. Magnetohydrodynamics 1987, 23, 31 .

(34) Pshenichnikov, A. F. J . Magn. Magn. Mater. 1995, 145, 319

(35) Hansen, J. P.; McDonald, I. R. Theory of Simple Liquids, 2nd ed.; Academic Press Ltd.: London, 1986. retained. I ntegration over someof theangular coordinates can be evaluated without assumptions, giving

$$
\begin{aligned}
& G(T, H)=\frac{1}{2 v_{c}} L^{2}(\alpha) \iiint \lambda \frac{d^{3}}{r_{12}{ }^{3}}\left(3 \cos ^{2} \theta_{r}-1\right) \times \\
& g_{0}\left(r_{12}\right) r_{12}^{2} d r_{12} d \theta_{r} d \phi_{r}+ \\
& \frac{1}{2 v_{c}} \frac{1}{3}\left\{\frac{6}{5}-\frac{6}{5} \frac{L(\alpha)}{\alpha}+\frac{9}{5}\left[\frac{L(\alpha)}{\alpha}\right]^{2}\right\} 4 \pi \int_{d}^{\infty}\left(\lambda \frac{d^{3}}{r_{12}{ }^{3}}\right)^{2} \times \\
& g_{0}\left(r_{12}\right) r_{12}^{2} d r_{12}
\end{aligned}
$$

where $g_{0}\left(r_{12}\right)$ is the radial distribution function and $L(\alpha)=\operatorname{coth}(\alpha)-\alpha^{-1}$ is the Langevin function.

It is important to note that integration of $\left(3 \cos ^{2} \theta_{r}-\right.$ 1 ) over the surface of a sphere gives $0 .{ }^{6}$ Because of this and the long-range $1 / r^{3}$ dependence of dipolar interaction, the only nonzero contributions to the integral can come from the outer regions of the system, where the sample shape can prohibit integration over all angles $\theta_{r}$ and $\phi_{r}$. $\mathrm{G}(\mathrm{T}, \mathrm{H})$ therefore depends on the macroscopic shape of the system; as in refs 4, 6, and 8 we consider a needleshaped sample here with the long axis parallel to the external field. And sinceg $g_{0}\left(r_{12}\right) \rightarrow 1$ as $r_{12} \rightarrow \infty$, theintegral does not depend on the actual structure of the colloidal dispersion. The second integral does depend on $g_{0}\left(r_{12}\right)$. Here, the low concentration approximation $g_{0}\left(r_{12}\right)=1$ for $r_{12} \geq d$ is used.

Under the given conditions, $\mathrm{G}(\mathrm{T}, \mathrm{H})$ becomes

$$
\mathrm{G}(\mathrm{T}, \mathrm{H})=4 \lambda \mathrm{L}^{2}(\alpha)+\frac{4}{3} \lambda^{2}\left[\frac{6}{5}-\frac{6}{5} \frac{\mathrm{L}(\alpha)}{\alpha}+\frac{9}{5}\left(\frac{\mathrm{L}(\alpha)}{\alpha}\right)^{2}\right]
$$

Theterm between brackets, which is between 1 and $6 / 5$ for all values of $\alpha$, is not included in ref 8 . Equations 10 and 12 can also be found by Taylor expanding $F_{\mathrm{DHS}}$ around $\lambda=0^{36}$ and neglecting dipolar interactions beyond the pair level.

From (10), the chemical potential and pressure can be derived

$$
\begin{gathered}
\frac{\mu_{\mathrm{c}}-\mu_{\mathrm{c}}{ }^{0}}{\mathrm{kT}}=\ln \frac{\phi_{\mathrm{c}}}{1-\phi_{\mathrm{c}}}+7 \frac{\phi_{\mathrm{c}}}{1-\phi_{\mathrm{c}}}+\frac{15}{2}\left(\frac{\phi_{\mathrm{c}}}{1-\phi_{\mathrm{c}}}\right)^{2}+ \\
3\left(\frac{\phi_{\mathrm{c}}}{1-\phi_{\mathrm{c}}}\right)^{3}-\frac{\phi_{\mathrm{p}}}{\mathrm{f}}\left(\frac{\mathrm{df}}{\mathrm{d} \phi}\right) \mathrm{q}^{-3}-\ln \frac{\sinh \alpha}{\alpha}-2 \phi_{\mathrm{c}} \mathrm{G}(\mathrm{T}, \mathrm{H}) \\
\frac{\mu_{\mathrm{p}}-\mu_{\mathrm{p}}{ }^{0}}{\mathrm{kT}}=\ln \frac{\phi_{\mathrm{p}}}{\mathrm{f}} \\
\frac{\mathrm{Pv_{ \textrm {c } }}}{\mathrm{kT}}=\frac{\phi_{\mathrm{c}}\left(1+\phi_{\mathrm{c}}+\phi_{\mathrm{c}}{ }^{2}\right)}{\left(1-\phi_{\mathrm{c}}\right)^{3}}+\frac{\phi_{\mathrm{p}}}{\mathrm{f}}\left(\mathrm{f}-\phi_{\mathrm{c}} \frac{\mathrm{df}}{\mathrm{d} \phi_{\mathrm{c}}}\right) \mathrm{q}^{-3}-
\end{gathered}
$$

Thehard-sphere parts in (13) and (15) aretaken from the scaled particle theory. ${ }^{26}$

According to (14), the volume fraction of polymer in the available volume, $\phi_{\mathrm{p}} / \mathrm{f}$, is the same for two phases in equilibrium. Finding the composition for two coexisting phases I and II therefore comes down to solving $\mu_{c}{ }^{\prime}\left(\phi_{c}{ }^{\prime}, \phi_{\mathrm{p}} / \mathrm{f}, \alpha\right)=\mu_{c}{ }^{\prime \prime}\left(\phi_{c}{ }^{\prime \prime}, \phi_{\mathrm{p}} / \mathrm{f}, \alpha\right)$ and $\mathrm{P}^{\prime}\left(\phi_{c}{ }^{\prime}, \phi_{\mathrm{p}} / \mathrm{f}, \alpha\right)=$ $\mathrm{P}^{\prime \prime}\left(\phi_{c}{ }^{\prime \prime}, \phi_{\mathrm{p}} / f, \alpha\right)$ at a given $\alpha$ and $\phi_{\mathrm{p}} / \mathrm{f}$. The true polymer concentration in each phase follows from the imposed $\phi_{\mathrm{p}} / \mathrm{f}$ and $\mathrm{f}$ for each phase.

(36) Barker, J . A.; Henderson, D. Rev. Mod. Phys. 1976, 48, 587. 

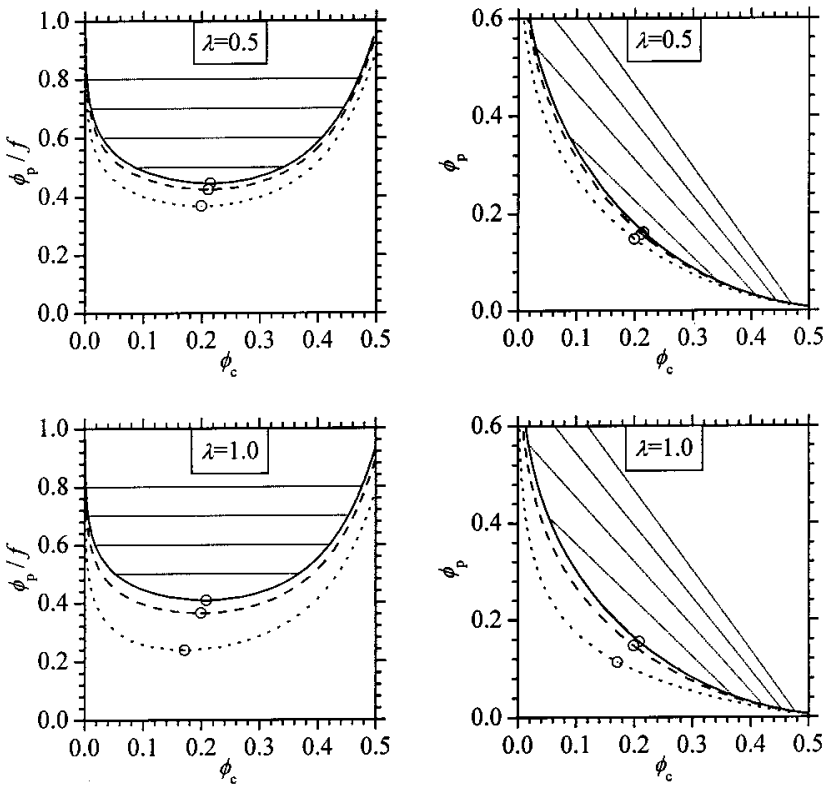

Figure 2. Gas-liquid binodals for a system of magnetic colloids + nonadsorbing polymer with $\mathrm{q}=0.5, \lambda=0.5$ (top) and $\lambda=1.0$ (bottom), and $\alpha=0$ (solid line), $\alpha=2$ (dashed line), and $\alpha=10^{6}$ (dotted line). Critical points areindicated by open circles; straight lines represent node lines.

\section{Results and Discussion}

3.1. Phase Diagrams. Phasediagrams werecalculated for a polymer/colloid size ratio $q=0.5$, a value that gives good agreement between the theoretically and experimentally ${ }^{24}$ found colloid concentration at thecritical point.

The most commonly used ferrofluid contains particles with a magnetite $\left(\mathrm{Fe}_{3} \mathrm{O}_{4}\right)$ core with a diameter $\mathrm{d}_{c}$ of typically $10 \mathrm{~nm}$, and an oleic acid shell to make them soluble in apolar solvents. ${ }^{1}$ Their magnetic moment is $\mathrm{m}=(\pi / 6) \mathrm{d}_{\mathrm{c}}{ }^{3} \mathrm{M}_{\mathrm{Fe}_{3} \mathrm{O}_{4}}$, where $\mathrm{M}_{\mathrm{Fe}_{3} \mathrm{O}_{4}}=4.8 \times 10^{5} \mathrm{~A} \mathrm{~m}^{-1}$ and is the bulk saturation magnetization of magnetite. Assuming an ol eicacid shell thickness of $2 \mathrm{~nm}$, an interaction parameter $\lambda=0.56$ is found, corresponding to a maximum magnetic attraction of $-1.1 \mathrm{kT}$ between two particles.

Figure 2 shows phase diagrams calculated for $\lambda=0.5$ and three different field strengths: $\alpha=0, \alpha=2$ (typically achievable by a small electromagnet), and $\alpha=10^{6}$ (approaching the situation of complete alignment). The destabilizing effect of a magnetic field is clear from Figure 2: at a given colloid concentration, a colloid-polymer mixture phase separates at lower polymer concentration in a field than it does in zero field. Over the entire $\phi_{c}$ range, phase instabilities can be found at roughly $7 \%$ $(\alpha=2)$ or $17 \%\left(\alpha=10^{6}\right)$ lower $\phi_{p}$.

Although the strength of dipolar interaction is significant when $\lambda=0.5$, its influence on the phase diagram is weaker than one might at first expect. The weak effect is due to the anisotropic nature of the dipolar interaction, which makes some configurations in the integration in (9) cancel each other. The difference with isotropic attraction can be illustrated by deriving the free energy for a system of spheres with isotropic mutual attraction, $\mathrm{u}=-\mathrm{C} / \mathrm{r}^{6}$, using perturbation theory. ${ }^{35}$ This yields

$$
\begin{aligned}
& F=F_{H S}-\frac{1}{2 v_{c}} N \phi \int_{0}^{\infty} \frac{C}{r^{6}} g_{0}\left(r_{12}\right) 4 \pi r_{12}^{2} d r_{12}= \\
& F_{H S}-12 N k T \phi \gamma
\end{aligned}
$$

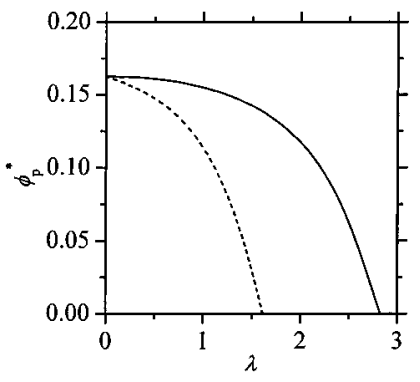

Figure 3. Polymer concentration $\phi_{\mathrm{p}}{ }^{*}$ at the critical point versus $\lambda$ for $q=0.5$ and $\alpha=0$ (solid line) or $\alpha=10^{6}$ (dashed line).

where $\gamma=\beta C / d^{3}$ and represents the attraction, relative to $\mathrm{kT}$, between particl es at closest distance. As in (11), the low concentration approximation $g_{0}\left(r_{12}\right)=1$ for $r_{12} \geq d$ was used in (16). N ow consider a simplified form of eq 10: if the magnetic moments are completely aligned $(L(\alpha)=$ 1 ) and $\lambda$ is small, the second term in (12) is negl igible and the correction to the hard spher efreeenergy is $-4 \mathrm{NkT} \phi \lambda$. This means that for the samestrength of pair interaction, the correction to the hard sphere free energy due to isotropic attraction is six times as high as the correction due to dipolar interaction (note again that $\lambda$ denotes half the maximum attraction).

Phase diagrams for $\lambda=1$ (Figure 2 ) are similar to those for $\lambda=0.5$, but the effect of a magnetic field is stronger: thelowering of thebinodal upon application of an external field is about $10 \%$ for $\alpha=2$ and $41 \%$ for $\alpha=10^{6}$.

3.2. Critical Point and Critical $\lambda$. The critical point, in particular $\phi_{p}$, depends on both the strength of the external field and the strength of dipole-dipole interaction. The shift of the critical point toward lower $\phi_{\mathrm{p}}$ upon increasing $\alpha$ and/or $\lambda$ was al ready evi dent from Figure 2 but is explicitly plotted as a function of $\lambda$ in Figure 3 . In zero field, magnetic interaction hardly influences the position of the critical point, e.g., at $\lambda=0.7, \phi_{\mathrm{p}}$ is lowered by merely $5 \%$. At (nearly) infinite field strength, the influence of dipolar interaction is much more pronounced: now, $\lambda=0.15$ al ready suffices to lower $\phi_{\mathrm{p}}$ by $5 \%$.

At $\phi_{\mathrm{p}}=0$, the critical points correspond to the critical points of pure dipolar hard sphere fluids. In the absence of a magnetic field, the critical value of $\lambda$ for isotropic gas-liquid coexistence according to perturbation theory is 2.83. However, the problem of whether dipolar hard sphere fluids can phase separate in zero field is still not resolved; in the past 30 years, theoretical and simulation results both in favor ${ }^{37-39}$ and against it ${ }^{9,40,41}$ have been reported. The result found with perturbation theory certainly does not clarify this question, because $\lambda$ is clearly out of the range where the expansions described in the theory section are valid.

At infinitefield strength and in the absence of polymer, the critical value of $\lambda$ for magnetized gas-liquid coexistence is 1.62. This value differs significantly and is much higher than the $\lambda>0.95$ for a magneticlatticegas model. ${ }^{5}$ It is, on the other hand, much lower than the limit of $\lambda>5.6$ found by Monte Carlo simulations ${ }^{42}$ It should be noted, however, that the aforementioned theories poorly

(37) Rushbrooke, G. S.; Stell, G.; Høye, J . S. Mol. Phys. 1973, 26, 1199

(38) Ng, K.-C.; Valleau, J .; Torrie, G.; Patey, G. Mol. Phys. 1979, 38, 781.

(39) Camp, P. J .; Shelley, J . C.; Patey, G. N. Phys. Rev. Lett. 2000, $84,115$.

(40) Caillol, J .-M. J . Chem. Phys. 1993, 98, 9835.

(41) Sear, R. P. Phys. Rev. Lett. 1996, 76, 2310.

(42) Stevens, M. J .; Grest, G. S. Phys. Rev. Lett. 1994, 72, 3686. 
describe the behavior of strongly interacting particles $(\lambda>1)$.

As $\lambda$ is about 0.5 for most magnetite-based ferrofluids, it is surprising that field-induced phase separation in these ferrofluids has been so often reported. ${ }^{12-16}$ Even a very high estimate of $\lambda$, taking a core diameter of $12 \mathrm{~nm}$, a surfactant thickness of $2 \mathrm{~nm}$, and neglecting the nonmagnetic surface layer, gives a $\lambda$ value of only 1.1. This is too small to explain the observed field-induced instabilities theoretically. Rather, these instabilities must be attributed to polydispersity, 43 the presence of small aggregates, ${ }^{44-46}$ van der Waals attraction, ${ }^{47}$ the influence of free oleic acid (dimers) which may act as a small depletion-inducing polymer, or a combination thereof. I ndeed, in our experimental study ${ }^{24}$ on the phasebehavior of a high-quality ferrofluid, containing negligible aggregates and free ol eic acid, we found no phaseseparation at any concentration and magnetic field strength. This is in agreement with the theoretical prediction.

In several papers, the described procedurefor preparing magnetic fluids does not include removal of unreacted oleic acid, thereby making the presence of free ol eic acid in thosesamples likely. If weconsi der freeol eicacid dimers (the main form in organic solvents) as a small polymer with a radi us of gyration of, say, $1 \mathrm{~nm}(q=1 / 7)$, thetheory described here predicts that a ferrofluid with a typical concentration of $\phi_{\mathrm{c}}=0.2$ destabilizes in zero field at $\phi_{\mathrm{p}}>$ 0.22 , which corresponds to an excess oleic acid concentration of only $58 \mathrm{~g} \mathrm{~L}^{-1}$. This indicates the importance of

(43) I vanov, A. O. Colloid J . 1995, 57, 321

(44) Charles, S. W. Chem. Eng. Commun, 1988, 67, 145

(45) Scholten, P. C. Chem. Eng. Commun. 1988, 67, 331.

(46) Buzmakov, V. M.; Pshenichnikov, A. F.J . Colloid I nterfaceSci. $1996,182,63$.

(47) Scholten, P. J . Magn. Magn. Mater. 1983, 39, 99. removing free oleic acid, or in fact any steric stabilizer, to obtain stable ferrofluids.

\section{Conclusions}

By use of a combination of the "free volume approach" for colloid-polymer mixtures and a perturbation theory for dipolar hard spheres, the phase behavior of mixtures of magnetic colloids and nonadsorbing polymers at several magnetic field strengths was investigated. The polymer induces an effective isotropic attraction between coll oids, the strength and range of which can be modified independently from magnetic interaction. A magnetic field modifies the dipolar interaction between colloids.

For colloids with magnetic interaction comparable to that in commonly used magnetite-based ferrofluids $(\lambda=$ 0.5 , maximum attraction $-1 \mathrm{kT}$ ), phasecoexistenceis only found in thepresence of polymer. Application of a magnetic field promotes phase separation, but even at infinite field strength the phase boundary shifts to only $17 \%$ lower polymer concentrations. For higher interaction strength $(\lambda=1.0$, maximumattraction $-2 \mathrm{kT})$ the binodal is shifted toapproximately $41 \%$ lower polymer concentrations. This strong dependence on $\lambda$ must be borne in mind when dealing with real ferrofluids, which may contain larger particles because of polydispersity.

Field-induced instability of ferrofluid without polymer was found only at $\lambda>1.62$, but the significance of this value is not without doubt, since the theory is formally only valid for $\lambda<1$.

The theory described here may hint at the effect of unreacted surfactant in, for instance, oleic acid stabilized ferrofluids. It was estimated that a concentration of $58 \mathrm{~g}$ $\mathrm{L}^{-1}$ oleic acid may al ready destabilize a ferrofluid with a volume fraction of $20 \%$.

LA011116R 